\title{
Intramedullary stabbing spinal cord injury: Perioperative optimized management for a successful surgical outcome
}

\begin{abstract}
We present a rare case of fully penetrating spinal cord injury in an 8-year-old girl who accidentally stabbed herself with a crochet needle at L4-L5 level. She presented to the local Accident and Emergency Department without neurological deficit. This is the full account of her perioperative management resulting in total neurological preservation.
\end{abstract}

Keywords: stabbing, spinal cord injury, intramedullary, perioperative management, intraspinal foreign body
Volume 10 Issue I - 2020

Pablo Martínez-Soler,' Juan F MartínezCanca $^{2}$

'Medical Student, University of Aberdeen, United Kingdom ${ }^{2}$ Consultant Neurosurgeon, CHIP Advanced Hospital Group, Spain

Correspondence: Dr Juan F Martínez-Canca, MD, MRCS, Consultant Neurosurgeon, Department of Neurosurgery, CHIP ADVANCED HOSPITAL GROUP, Avda Carlos de Haya I2I, Málaga 29010, Spain, Tel +34653819369, Fax +3495101 II 150, Email neurosurgery@europe.com

Received: December 26, 2019 | Published: January 16, 2020

\section{Introduction}

Whilst gunshot and missile penetrating spinal trauma is well described in the medical literature in connection with military environment, accidental and even crime-related penetrating spinal cord injuries are relatively uncommon clinical scenarios that normally lead to permanent neurological deficit as well as relevant socioeconomic implications for the affected patient and families. There seems to be no formal consensus in the appropriate comprehensive management of these situations.

This is the case of an eight-year-old girl who was playing and jumped backwards falling onto a sofa where several crochet needles had been left naked. She sustained a dramatic single fully penetrating injury to her spine.

\section{Case report}

A previously fit and well, eight-year-old girl was taken by ambulance to the local Emergency Room with a penetrating spinal injury, accidentally sustained at home whilst playing with her brother. She jumped backwards onto a sofa where several crochet needles had been left unprotected, stabbing herself at the left side of L4-L5 level. ${ }^{1-3}$ Only the tip of a $22 \mathrm{~cm}$ ( 8.5 inches) crochet needle was just visible over her left flank through a clean bloodless puncture entry point. Initial inspection ruled out active haemorrhage and CSF leak. She was neurologically intact, and no other injuries were elicited on examination. The patient appeared to be comfortable in her spontaneous prone position since the time of the event, approximately thirty minutes earlier. Following adequate immobilisation (patient remained prone) and primary survey, she was taken to the Radiology Department where computed tomography (CT) was obtained (Figure 1).
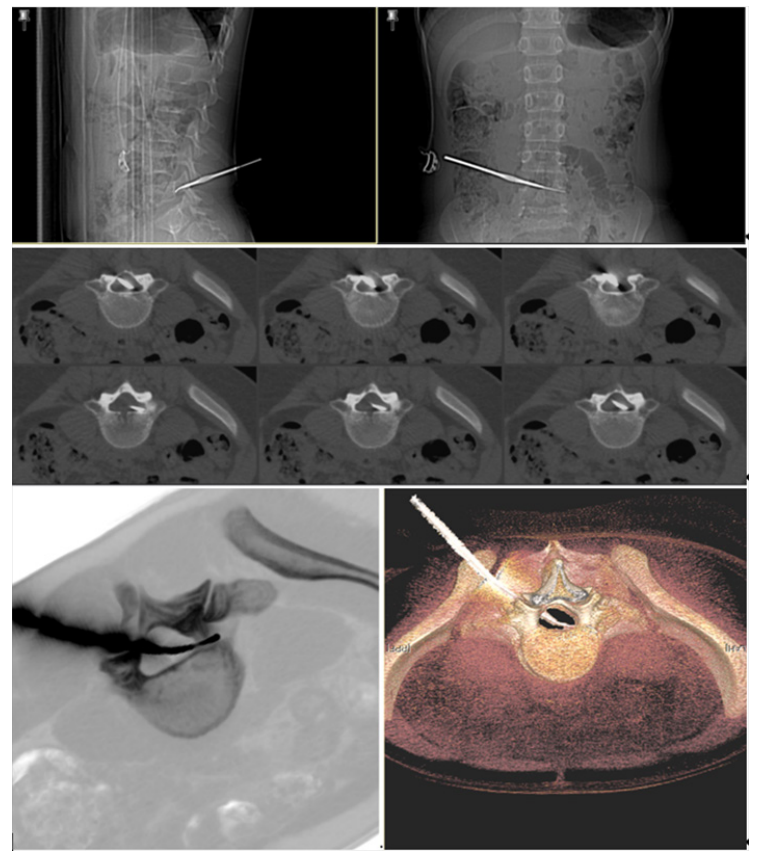

Figure I CT and 3-D CT images demonstrating the anatomical trajectory and positioning of the penetrating foreign body at the level of L4-L5.

Images demonstrated a transecting foreign body in keeping with a crochet needle penetrating the soft tissue from her left flank, disrupting the facet joints at the left L4-L5 motion segment, fully traversing the spinal canal, and reaching the posterior aspect of L4 vertebral body just lateral to the right pedicle. Decision was made to take her to the operating room. ${ }^{4-6}$ 
Intubation was performed in prone position and paediatric dose of intravenous broad-spectrum antibiotic was administered on induction. As full vaccination calendar was up to date, no additional coverage was needed. Full neuromonitoring including motor and somatosensory evoked potentials was established. Standard draping was applied to the operating field and no electrocoagulation was used for the procedure in prevention of potential collateral spinal cord lesion. A midline incision was performed at L4-L5 level and standard dissection in anatomical layers carefully conducted, following the ventral trajectory of the foreign body. Fortunately, an interlaminar approach was sufficient to visualize the full anatomical extent of the problem without the need to perform any bony dissection at all. Shockingly, the operative findings demonstrated only a minor disruption at the inferior aspect of the left L4-L5 facet joints and an intact dural sac astoundingly escaping any penetration whilst letting the foreign body to pass by harmlessly through the posterior region of the spinal canal, quite contrary to what we had clearly expected in the light of preoperative imaging. ${ }^{7,8}$ Once the tip of the foreign body was identified lying adjacent to the right pedicle in the vicinity of the posterior aspect of L4 vertebral body, soothing mechanical extraction was performed, maintaining the integrity of the whole anatomy. Standard surgical closure was then applied. Neuromonitoring remained within normal parameters with no registered abnormalities throughout the procedure. The patient woke up uneventfully from her general anaesthesia and neurological examination confirmed absence of any deficit post-operatively. ${ }^{9}$

\section{Conclusion}

This is an excellent example of effective consensual interdisciplinary management of a complex case from the time of attendance to the local Emergency Room, involving ED physicians, paediatricians, neurosurgeons, radiologists, neurophysiologists, and microbiologists working together with a perfect understanding of a potentially critical situation. Coordination and fast teamwork were the keys to coordinate a sequence of essential specialist input in a timely fashion. Maintaining alert awareness based on the result of promptly obtained radiological images and establishing clear interdisciplinary clinical priorities allowed a flawless resolution of this case. The almost miraculous outcome provided the happiest possible ending and the patient was discharged home in 24 hours.

From a clinical viewpoint, we are delighted to report that, even in the light of dramatic preoperative CT images such as those illustrating this case report, intramedullary lesion does not have to necessarily exist. Having said that, we emphasize that strict observance of the established surgical and perioperative protocols must be meticulously followed to the point, in order to prevent possible inadvertent and/or added neurological insult.

\section{Acknowledgments}

None.

\section{Conflicts of interest}

The authors declare no conflicts of interest.

\section{References}

1. Baghai P, Sheptak PE. Penetrating spinal injury by a glass fragment. Neurosurgery. 1982;11(3):419-422.

2. Prasad BC, Vemula RC, Varaprasad G. Nonmissile Penetrating Spinal Injury with an Impaled Knife: Case Report. Indian J Surg. 2010;75(3):237-238.

3. Shahlaie K, Chang DJ, Anderson JT. Nonmissile penetrating spinal injury. Case report and review of the literature. J Neurosurg Spine. 2006;4(5):400-408.

4. Tian D, Zhu B, Shi-jia Z. Pediatric Penetrating Spinal Canal Injury by Glass Cutting caused Cauda Equina Syndrome: Case Report and Literature Review. Journal of Pregnancy and Child Health. 2015;2(6):205.

5. Collins A, Moriarty J, Laing A, et al. Penetrating spinal injury by a projectile diamond bead in a limestone quarry. Injury Extra. 2008;39(2):62-64.

6. Gopal V, Mahadevan K. Penetrating Thoracic Spinal Injury with Impacted Glass Fragment. The Journal of Spinal Surgery. 2014;1:169-173.

7. Ye T, Jia L, Chen A, et al. Brown-Séquard syndrome due to penetrating injury by an iron fence point. Spinal Cord. 2009;48(7):582-584.

8. Gulamhuseinwala N, Terris J. Evolving presentation of spinal canal penetrating injury. Injury. 2004;35(9):948-949.

9. Connell RA, Graham CA, Munro PT. Is spinal immobilisation necessary for all patients sustaining isolated penetrating trauma. Injury, 2003;34(12):912-914. 